BOOK REVIEW

\title{
Cace Sorin, Fitzek Sebastian (Eds.): ICCV 2020 SOCIAL REPORT. COVID19 IN ROMANIA, DATA, ANALYSIS, EVOLUTIONS AND STATISTICS, Bucharest: Romanian Academy, 2020, p.273
}

\section{Sebastian FITZEK ${ }^{4}$}

DOI: 10.35782/JCPP.2020.3.06

In less than six months of laborious research, the Quality of Life Research Institute has managed to print a complex and interesting collective volume, with latest data, analysis, evolutions and statistics that focused on Romania in 2020. The shock of its massive spread and the unexpected rise of the Covid19 virus around the world have generated a number of fundamental questions and analysis for the entire world of scientific community. Through dedication to the subject, the Quality of Life Research Institute team retaliates via 6 chapters the quality of life evolution during the first months of the year, the social policies of health elaboration and social protection, the demographic changes and the standard of living for the Romanian citizens which has undergone sudden changes, unpredictable for any scientific forecast. The demeanour of the whole phenomenon and its evolutionary stages are abundantly reflected in the 6 studies elaborated in a structure determined by the broad preoccupations of the institute, but also by the reason of some reactions in fields such as health, economy, education, demography and social assistance. Numerous fastidious important aspects are missing today from the Romanian public debate. In this account, the volume offers a refined picture of a series of analysis, arguments and specialized statistics that scientifically substantiate the authors' statements.

From the very beginning, in the first chapter, the authors Cătălin Zamfir (Scientific Researcher 1 and Director of the Quality of Life Research Institute) and Elena Zamfir

\footnotetext{
${ }^{4}$ Senior Researcher The Research Institute for Quality of Life, Romanian Academy and Lecturer at Faculty of Communication and Public Relations, National University for Political Studies and Public Administration, Bucharest, email: sebastian.fitzek@comunicare.ro
} 
(Scientific Researcher 1) display the issue of the pandemic, from a synthetic perspective, regarding to its imminent impact on the quality of life over an especially short, irreversible and surprising period of time in its exponential motion and evolution. In order to ascertain this exponential growth rate, the two authors capture the entire phenomenon in four successive hypostases of time: the pre-crisis period with all the anxieties reported, then, only throughout the press, the period of emergency and the relaxation period. The debated charts and data compare the situation from Romania with the situation from other European countries, noting behind these figures, the distinct immediate social effects of the crisis, but also the significant changes in human relations and quality of life. From the same perspective, the immediate effects that unfolded in society through physical distancing, home office, isolation, solidarity and defiance to the recommendations of the authorities are analysed along with major processes that have decisively changed relations either socially, interpersonally, institutionally or professionally.

The message of the authorities to stay at home for as long as possible was differently perceived by the population. Also the constraint of the fundamental right to move during the emergency state, especially for the elderly, generated a series of measures unprecedented throughout post-December history. The impact of the crisis on the quality of life paradigm is another important segment that the authors assess in a sociological analysis extended to the development of social uncertainty, of hereafter increasing insecurity and especially by declining of confidence in state authorities, effects that were complied with the professional level of Romanian citizens. The population's reaction to the crisis was analysed by monitoring the main institutes of national statistics. Thus, the data presented show a series of communication issues and dilemmas that the Romanian State has managed with difficulty.

The second chapter brings in the essence of quality of life as main subject for the standard of living in Romania under the siege of COVID19. The researchers: Daniel Arpinte, Sorin Cace, Simona Mihaiu, Iulian Stănescu, Simona Maria Stănescu and Sebastian T,oc display an X-ray of the field data in relation to the social protection measures and policies bore by the Government through the Emergency Ordinances. The accuracy and collection of data in record time, exposed this chapter to a series of analysis and number crunching that apparently have no direct links, but the authors were able to consequentially mount and unravel them in a facile manner. Along with other countries, the "lockdown" effect pushes Romania towards a record of negative figures, and the most vulnerable become the Romanian citizens who want a working place to return home from. Presenting these data and situations compared to other states, the authors propose a series of policies that guide and improve the standard of living of individuals. Research shows how economic and health problems directly affect all other aspects of life. The lessons of the crises that Romania went through with an impact on the population's income indicate the urgent need for state intervention and, 
implicitly, the increase of the costs related to social protection. The issues raised are worrying, but do not go unanswered, with the authors bringing a number of viable recommendations and options to possible directions for action. The lessons of the crises that Romania went through with an impact on the population's income indicate the urgent need for state intervention and, implicitly, the increase of the costs related to social protection. The issues raised are worrying, but do not go unanswered, with the authors bringing a number of viable recommendations and options to feasible directions for action.

In the third chapter, the authors Mălina Voicu, Daniel Arpinte, Delia Bădoi, Alexandra Deliu, Simona Mihaiu, Simona Maria Stănescu and Cristina Tomescu analysed the evolution and demographic trends in Romania during the pandemic. The health crisis has severely affected all age groups, especially areas with social and economic vulnerability. Mortality dynamics, fertility and migration have generated a series of state measures nonetheless insufficient to fight the negative effects at the national level. The stated hypotheses were built on what happened, in the same domains at European level. Thus, the authors offer a series of answers and corrective actions in the hope of better results to those currently found. The merit of this chapter is also found in the dissemination of valuable information, which the authors extracted from a huge volume of data collected during months of research.

In the fourth chapter, the authors Bogdan Florian and Sebastian T,oc evaluate the education system in Romania during the pandemic. The authors' answers provide clarity, vision and qualitative information in the field of reform policies. The consistency of the included analysis was prioritized on the following dimensions of the current challenges of Romanian education: inequality of opportunities in education; digital skills and the transition to the online teaching-learning system; sanitary conditions in schools; recommencement or completion of the school year at European level. The identification of the most sensitive issues is followed by an interesting series of public policies, which the authors propose as active measures to resolve crises in the education system.

In the fifth chapter, the authors Mariana Stanciu, Adina Mihăilescu and Cristina Humă analysed the risks of the food crisis due to the pandemic at both national and international level. The medical crisis has spawned the food crisis, but there are other factors, as the authors point out, that are exacerbating the global food crisis. Drought, some inappropriate government policies, fragility of supply chains due to traffic restrictions, export bans and, above all border closure policies have had a major impact on this sector, implicitly on the quality of life of each individual. Having this background, the food crisis is confirmed by key indicators that have influenced the consumption of the population before and after the onset of the pandemic. The recording of differences in figures between 2019 and 2020 shows why incomes and 
purchasing power are uncertain, favoring social inequality between high and low incomes. Rising unemployment is another key factor in lowering living standards, especially in the field of private employers. Emigrants' return, the reduction of population mobility, poverty, the evolution of household incomes and the food consumption basket are some of the indicators brought out by the figures in a unitary photograph in which we find an everyday reality with many unsettled problems.

In the sixth chapter, Professor Vasile Morar contributes with a philosophical vision on the moral and ethical impact that pandemics have had in human history compared to the current situation. The necessity to rethink the entire education project is, in the author's opinion, the cornerstone for changing attitudes and behaviours in crisis situations, and in their absence, humanity could go through the most acute crisis, the moral one.

Interpersonal friendship is the path that saved man from the most difficult situations and made him survive any weather, wars, plagues and cataclysms. Natural selection is also a selection of friendships, where man chooses a correct system, true or false or based on deceit. Restoring the links between man and nature is a correct choice, but it must be rethought by each individual, community and state, otherwise the preservation of humanity as a natural element can be endangered.

The entire volume has a wide addressability and is not limited to the academic environment. Its structure was organized in a series of topics, problems and solutions in the form of a handbook, a guide for how to avoid in such a period the risks of poor decisions. Beyond its sententious side, the most informed public of this study is represented by the political decision makers who have the power to manage either effectively or inappropriately. The social, economic and especially human radiography of this book will soon be found in a new historical lesson, important for those responsible for decisions, but also a guide to remember for future generations of tomorrow's Romania. 